\title{
La laïcité et l'école de la République
}

\section{Guy Coq}

\section{OpenEdition}

Journals

Édition électronique

URL : http://journals.openedition.org/trema/2747

DOI : $10.4000 /$ trema. 2747

ISSN : 2107-0997

\section{Éditeur}

Faculté d'Éducation de l'université de Montpellier

\section{Édition imprimée}

Date de publication : 1 avril 2012

Pagination : 144 - 164

ISSN : 1167-315X

\section{Référence électronique}

Guy Coq, «La laïcité et l'école de la République », Tréma [En ligne], 37 | 2012, mis en ligne le 01 avril 2014, consulté le 02 mai 2019. URL : http://journals.openedition.org/trema/2747 ; DOI : 10.4000/ trema. 2747

Ce document a été généré automatiquement le 2 mai 2019.

Trema 


\section{La laïcité et l'école de la République}

\section{Guy Coq}

1 Dans la présentation de la laïcité on met constamment et à juste raison la loi de 1905 au premier plan. À travers quelques articles souvent commentés et que l'on devrait faire apprendre par cœur aux enfants des écoles, l'essentiel est dit: le rappel de la liberté de conscience (article 1), la garantie du libre exercice des cultes, qui est une liberté publique, collective (article1). Puis l'article 2 objet de tant de contresens, car si la république ne reconnait plus certains cultes au sens où le concordat napoléonien qu'elle abroge le voulait, bien entendu, une fois séparée des cultes, il faut bien que la République dialogue avec les représentants des cultes, et les reconnaisse comme responsables. Et puis, à l'article 4 se précise que la loi veut une liberté réciproque: la République est indépendante de toute confession religieuse, mais de l'autre côté, les confessions sont libres de s'organiser de manière autonome puisque les associations qui génèrent les cultes le font «en se conformant aux règles d'organisation générale du culte dont elles se proposent d'assumer l'exercice »...

On peut aussi prendre la question d'un point de vue chronologique en distinguant trois grands piliers dans l'instauration de la laïcité. Le premier se situe le 13 avril 1790 quand l'assemblée ne reconnait plus le catholicisme comme religion d'État, c'est un acte décisif qui ne sera plus remis en cause puisque même Napoléon ne le fait pas. Avec l'empereur, le catholicisme est reconnu comme religion de la majorité des français et la religion devient service public.

Le second pilier est la séparation de l'école et de l'église, acte dont nous explorerons la place centrale dans la laïcité. Et, confortée par la laïcité de l'école, la République posera en 1905 la séparation des églises et de l'État. Enfin, on peut exposer la laïcité selon la logique des divers plans où elle s'exerce. On peut ainsi discerner trois niveaux. Au premier niveau la laïcité apparaît comme synonyme de neutralité. Du moins est-ce ainsi que se la représente la majorité des français. Être laïque, c'est être neutre du point de vue de la religion. En s'arrêtant à cette définition, on réduit terriblement le sens du principe de laïcité. Mais il est vrai, on l'a vu, que la laïcité implique la neutralité religieuse de l'État, ce qui laisse la possibilité qu'il ne soit pas neutre sur d'autres sujets. Mais cette 
neutralité ouvre la société à l'accueil du pluriel sur le plan religieux, et finalement de toutes les options philosophiques concernant les fins ultimes.

Cependant, il faut fonder cette société accueillante à la diversité et le principe de neutralité de l'État, et ici s'annonce un deuxième plan: la laïcité comme incluant des valeurs. Il y a toute une configuration de valeurs éthiques sur lesquelles se fonde la laïcité : la tolérance, mais aussi l'accueil de l'autre, le sens de l'humanité, la liberté comme valeur, la dignité de la personne. En fait la notion même de valeur, au niveau où se distinguent les options métaphysiques, est interne à la laïcité. C'est pourquoi la question des valeurs communes est vitale pour éclairer le principe de laïcité.

Mais il y a un troisième registre de la laïcité qui serait mutilée si on la limitait à l'aspect éthique : la laïcité est un principe instituant de l'ordre social. Elle dit la caractéristique d'une société qui n'accepte plus de dépendre d'une altérité divine, d'une religion pour s'instituer. L'ensemble de la société est remis à l'humanité elle-même, à la responsabilité des citoyens, c'est pourquoi la laïcité comme principe d'autonomie de la société débouche sur le principe démocratique.

Or, le deuxième plan, celui de l'éthique a été historiquement établi lors de la séparation de l'école et de l'Église. Et c'est bien dans cette étape qu'apparaît l'importance du domaine éthique pour la laïcité. En ce sens, la laïcité de l'école est un aspect essentiel de la laïcité. Il convient d'insister sur ce point. L'école de la République n'est pas un simple espace d'application du principe de laïcité, elle est un lien où s'explicite une part du sens même du principe de laïcité.

\section{Retour sur la fondation de la laïcité scolaire}

6 Dans la présentation de la laïcité, il y a indiscutablement une tendance très répandue à concentrer l'attention exclusivement sur la loi de 1905, à oublier que cette séparation des églises et de l'État fut précédée par une séparation de l'école et de l'Église que représente la fondation de l'école laïque. Rappelons les deux lois portées par Jules FERRY, 16 juin 1881 : gratuité de l'école et 28 mars 1882 : obligation et laïcité de l'école par, notamment, le déplacement de l'instruction religieuse hors de l'école à l'église, et par l'établissement d'une éducation morale. Enfin la laïcisation du personnel de l'enseignement est instituée par la loi GOBLET du 30 octobre 1886.

7 Le fait qu'historiquement la laïcité commence par l'école peut en éclairer le sens: la République est toute neuve. Et il est clair que l'Église exerce un pouvoir qui dépasse largement son sens spirituel : elle répand des idées politiques qui agissent contre l'idée républicaine. Les républicains » ne peuvent admettre que les catholiques continuent à élever dans la condamnation de l'esprit moderne et des principes de 1789 toute une partie de la jeunesse. «Le catholicisme n'est pas seulement une religion : c'est aussi, à l'époque, une doctrine politique et sociale » (PROST, 1968).

Bref, on peut parler d'un lien congénital entre la laïcité scolaire et la République. C'est là, dans la naissance de la République, que la laïcité s'impose, comme condition essentielle. Mais en même temps le lien avec une éducation morale apparait d'emblée comme nécessaire à l'école laïque. Ferdinand BUISson écrit dès 1883 que si l'enseignement primaire se limitait au "lire, écrire, compter " sans aborder les questions morales et religieuses « ce serait fait de l'école laïque ${ }^{1}$ ». De même en 1908 un des artisans de l'école laïque, Gabriel COMPAYRÉ, "rappelle qu'en 1882 l'école laïque est un engagement 
d'honneur " à l'égard des parents, celui d'enseigner la morale aux enfants. Si cet engagement n'était pas tenu « ce serait la faillite de l'école publique » (OGNIER, 1994).

L'importance attachée à l'éducation morale apparait dès les programmes de juillet 1882 . Ceux-ci ont trois parties : 1) Éducation physique et préparation à la vie professionnelle ; 2) Éducation intellectuelle ; 3) Éducation morale.

10 La morale est un des trois piliers de l'enseignement. Elle comporte une dimension religieuse indépendante de toute confession, ce qu'on a souvent résumé en parlant des «devoirs de Dieu ». L'expression se trouve dans le programme du cours moyen (9 à 11 ans). Dans le programme du cours élémentaire, il s'agit «d'élever par exemple au sentiment d'admiration pour l'ordre universel et au sentiment religieux ». Au cours supérieur, la loi morale est ce à quoi « il ne peut faillir sans se sentir coupable envers luimême et envers Dieu ».

11 Cette dimension religieuse ne devrait pas occulter aujourd'hui la qualité de la pensée éthique des pages consacrées à « l'objet de l'enseignement moral». On insiste sur le fait que celui-ci «se distingue de l'enseignement religieux sans le contredire ». L'objectif est « un apprentissage de la vie morale». Il s'agit aussi de développer une culture sur les "notions essentielles de moralité humaine communes à toutes les doctrines et nécessaires à tous les hommes civilisés ». Il n'y a pas à enseigner une morale théorique : «À l'école primaire surtout ce n'est pas une science, c'est un art, l'art d'incliner la volonté libre vers le bien ». L'initiation à la dignité personnelle de chacun est au premier plan des objectifs, avec l'éveil au sens de la responsabilité personnelle. Dans la page sur la méthode, certaines expressions sont significatives: faire éclore des sentiments vrais, toucher son cœur, "faire ressentir par une expérience directe la majesté de la loi morale », il s'agit de "former chez l'enfant le sens moral ». À tous les niveaux, l'insistance est sur les exemples, l'application, les exercices pratiques. Ce même souci du concret se retrouve dans un texte célèbre: la lettre de Jules FERRY à « Monsieur l'instituteur » à l'occasion de la seconde rentrée scolaire appliquant la loi du 28 mars $1882^{2}$. Quant à la répartition des tâches, Jules FERRY est clair: "L'institution religieuse appartient aux familles et à l'Église, l'institution morale à l'école ». Il insiste sur le rôle d'exemple que doit assumer l'instituteur et sur l'importance de l'éducation morale : « En vous dispensant de l'enseignement religieux, on n'a pas songé à vous décharger de l'enseignement moral, c'eût été vous enlever ce qui fait la dignité de votre profession. ».

12 L'aspect pratique est partout présent dans ce texte : «Il ne s'agit plus là d'une série de vérités à démontrer, mais ce qui est tout autrement laborieux, d'une longue suite d'influences morales à exercer sur de jeunes êtres, à force de patience, de fermeté, de douceur, d'élévation dans la caractère et de puissance persuasive ». En soulignant l'importance de l'éducation morale, Jules FERRY en marque aussi la difficulté : éviter d'un côté le verbiage, de l'autre d'empiéter sur le domaine du prêtre ou du pasteur.

Dans un travail quasi classique, Jean вAUBÉROT étudie les thèmes et les problèmes de la morale laïque entre 1882 et 1918 en examinant plus de 200 cahiers d'écoliers de la période (BAUBÉROT, 1997). Ces documents permettent de mettre en lumière d'une part les leçons dictées par les instituteurs, d'autre part les exercices et devoirs effectués par les enfants. Par rapport aux belles intentions des fondateurs, on observe une grande fidélité et une mise en œuvre remarquable.

Dans les écrits des fondateurs de la laïcité scolaire apparait l'extrême importance que la République attache à l'école. Ce soutien ferme des institutions politiques fait de l'école 
publique une institution forte qui illustre la grandeur de l'idée républicaine, qui porte la promesse du progrès et des Lumières. L'élan de la Révolution travaille cette image de l'école. Celle-ci repose sur un pilier solide : l'instituteur dont le prestige est immense.

\section{Une déconstruction de la laïcité scolaire?}

Cette présentation des fondations de l'école et de la laïcité scolaire est certes succincte. C'est au moins un rappel qui permettra de s'interroger sur les conditions d'une revitalisation de l'école de la République. Car, c'est un fait qu'il convient d'interroger, on a assisté dans la seconde moitié du XXe siècle à une longue déconstruction de la laïcité scolaire et en même temps de l'institution.

Les notions d'institution, en général, et d'institution éducative en particulier sont souvent trop peu élaborées. L'institution est une structure qui a un rôle médiateur entre l'individu et la société globale : elle intègre à celle-ci. Mais quand on parle d'institution, on vise une forme qui précède l'individu, que celui-ci ne crée pas par décision de s'associer. Les institutions sont portées par des finalités sociales : éduquer, rendre la justice, assumer la bonne gestion des services essentiels. Dans le cas de l'école, la finalité est d'éduquer: c'est-à-dire de permettre l'entrée dans une culture, et ainsi de se construire comme personne, et de devenir capable d'être un membre à part entière de la société. L'école publique tire fondamentalement sa légitimité de son articulation avec la République : à travers elle l'individu devient censément citoyen.

Comme institution, l'école répartit des rôles (le maître, l'élève) qui sont codés par des règles, des usages. Porteuse de l'ambition de transmettre une culture, elle a de ce fait la légitimité pour exercer une autorité.

Nul n'a mieux que Hannah ARENDT analysé ce lien entre l'autorité et l'éducateur et l'obligation pour lui d'aider l'enfant à entrer dans un monde qui le précède. «L'éducation est le point où se décide si nous aimons assez le monde pour en assumer la responsabilité et, de plus, le sauver de cette ruine qui serait inévitable sans ce renouvellement et sans cette arrivée de jeunes et nouveaux venus. C'est également avec l'éducation que nous décidons si nous aimons assez nos enfants pour ne pas les rejeter de notre monde, ni les abandonner à eux-mêmes, ni leur enlever leur chance d'entreprendre quelques chose de neuf, quelque chose que nous n'avions pas prévu, mais les préparer d'avance à la tâche de renouveler un monde commun" (ARENDT, 2007 [1968], p. 251-252). Or Hannah ARENDT articule fortement cette responsabilité du monde vis-à-vis de l'enfant et de l'autorité : «Dans le cas de l'éducation la responsabilité du monde prend la forme de l'autorité » ( ARENDT, 2007 [1968], p. 243). Dans une institution en bonne santé, l'autorité n'a besoin « ni de moyens extérieurs de coercition » ni d'avoir recours à des arguments pour se justifier, telle est l'analyse que développe Hannah ARENDT.

Décrire ainsi l'institution scolaire, c'est tout simplement reprendre les traits principaux qui l'ont caractérisée pendant des générations.

\section{1. Le mépris de la culture scolaire}

20 De même, il faudrait analyser ce qui caractérisait la culture scolaire. Une certaine tradition consistait à désigner ainsi ce dont l'école devait assurer la transmission. À côté des acquisitions fondamentales (lire, compter...), l'école est censée proposer une part de 
la culture héritée, celle que par tradition on considère comme capable de construire l'humanité à venir. On inclura dans cette culture scolaire des œuvres du passé, arts, textes à travers lesquels une humanité s'est construite. Au fond, l'idée est qu'en fréquentant des œuvres où des humains ont conquis leur humanité, on peut apprendre sa propre humanité.

De plus, une relation vive avec le passé est nécessaire pour élaborer le présent. Celui-ci ne se limite pas à la seule actualité. Il suppose de s'inscrire dans un devenir, dans une durée parfois longue. Mais cette relation avec du passé n'est aucunement une identification au passé. L'être humain individuel ou collectif s'identifie dans une relation neuve et originale avec des éléments du passé. Le présent se construit ainsi dans une relation avec l'ancien. Cette capacité de se situer dans un devenir individuel ou collectif permet de voir au-delà du présent un avenir possible. Les attitudes culturelles qu'on vient d'évoquer allaient de soi dans la culture scolaire. De même on admettait que le passé n'était pas automatiquement inférieur au présent, que des œuvres anciennes n'étaient pas dévalorisées de par leur seule antiquité.

\section{2. École laïque impossible?}

Or, aussi bien du côté des conditions de possibilité d'une institution éducative que du côté des conditions de légitimation d'une culture scolaire, on a assisté à de redoutables remises en cause, de sorte que la question s'est posée de la possibilité même d'une école laïque. Pour radicaliser le propos, osons: la démocratie a-t-elle rendu l'éducation impossible ? La République, telle qu'elle a évolué n'a-t-elle pas sapé ce fondement qu'était pour elle l'école laïque?

En ce qui concerne l'école comme institution, c'est à une véritable délégitimation qu'on a pu assister depuis le dernier tiers du XXe siècle. Il faudra mesurer un jour l'onde de choc de certains aspects de ce qui a suivi mai 68 . Ainsi, c'est un fait que dans cette période la culture spontanée de l'individu démocratique a développé certains excès: comment obtenir un consentement à l'école chez quelqu'un qui voudrait ne dépendre de rien de ce qui l'a précédé ? Qui voudrait que tout ce qu'il est, il en soit l'auteur ? On l'a vu, éduquer, c'est faire entrer dans une culture, dans un monde déjà là, que je n'ai pas choisi. Comment éduquer un individu pour qui toute obligation n'est que contrainte, toute contrainte n'est finalement que violence ? Où va-t-on alors? Car même une société démocratique ne peut se passer d'autorité et, plus, d'autorité institutionnelle.

24 Comment éduquer à la citoyenneté un être qui croit qu'il n'y a que des libertés individuelles, que sa liberté n'implique jamais autrui? L'auteur de ces lignes a souvent donné à réfléchir aux élèves de terminale des sujets qui questionnaient sur : rapport liberté / autrui, rapport liberté / société. Une posture idéologique très dure se manifestait alors : autrui est toujours par nature une limite à ma liberté. La société est la mort de la liberté. Que ma liberté puisse s'accroitre de coopérer avec la liberté des autres, est-ce un point de vue inaccessible? Qu'il y ait des libertés que je ne puis exercer que si autrui aussi désire les exercer, est-ce autre chose que du bon sens ? Si je suis seul à désirer la liberté de la presse, elle n'existera pas. Et puis il y a des libertés qu'on ne peut exercer qu'en commun, par exemple une décision qui transforme une ville. Et si elle ne s'exerce pas avec autrui, alors ma liberté est perdue, tout seul je suis moins libre que dans l'action avec autrui. Comment faire un citoyen de quelqu'un qui ne connaît la liberté que comme une propriété individuelle? Bien entendu cet individu extrême que j'évoque ici n'existe 
pas complètement. L'exemple sert à montrer des tendances qui rendent problématique la possibilité d'une école, d'une éducation.

Ajoutons que l'attitude consumériste vis-à-vis de l'école, qui tend à faire des familles de simples consommateurs d'école, les porte à oublier que sans le respect et la reconnaissance qu'elles expriment vis-à-vis de l'école, celle-ci ne parvient pas à se tenir, comme institution éducative. Elle ne se maintient que par la qualité des représentations d'elle-même qui circulent.

Les conditions de possibilité d'une culture scolaire véritable ont été remises en cause depuis le milieu du XXe siècle. Que la culture scolaire ait été dans l'histoire monopolisée par la bourgeoisie, c'est un fait. Mais cette culture générale véhiculée par le lycée, avec ses disciplines scolaires, a subi pendant des générations les attaques des réformateurs qui la rejetaient comme la «culture bourgeoise ». Que de livres pour dénoncer cette culture scolaire comme porteuse des idéologies illusionnant les classes populaires sur leur destin, comme légitimant l'élite des privilégiés... Que de propos faciles et chez de grands esprits pour dénoncer le « saucissonnage » des disciplines !

Nous l'évoquions plus haut: par-delà la lettre de ce qu'elle contenait (ou contient) la culture scolaire initie à des postures culturelles : par rapport au sens du temps, au bon usage des héritages. Les récits de la nation pouvaient inclure du légendaire, n'empêche, comme récits, ils invitaient l'individu à prendre position dans le temps et, sans le dire, ils initiaient à assumer la question des repères individuels et collectifs. On a critiqué la mémorisation des dates, en histoire, même lorsqu'on s'est avisé que ce travail de chronologie avait un effet utile qu'il était seul à produire : former la conscience de la temporalité.

La culture scolaire diffusait une haute idée de la culture comme construction d'une humanité. Elle n'était pas aplatie sur la quête immédiate d'une utilité sociale. Or là encore, on peut détecter une tendance destructrice de la culture scolaire, une perte de la finalité humaniste.

29 La crise institutionnelle et culturelle de l'école s'est concentrée sur le collège. Celui-ci devint à travers les réformes le dernier palier de l'école obligatoire pour tous. Mais en même temps, et inévitablement, il est une étape intermédiaire, un lieu où finalement s'opère un tri, osons le mot, une sélection vers des études longues. C'est un fait souvent négligé : le système scolaire en démocratie est travaillé par deux logiques sociales. D’un côté, il est soumis à une logique égalitaire : donner à tous les connaissances, la culture qui leur permettra de s'intégrer comme citoyens dans la démocratie. Cette logique valorise les efforts permettant à tous d'atteindre à ces aptitudes jugées indispensables. Mais d'un autre côté la société démocratique fait porter sur l'école une logique élitaire: cette société ne peut pas se passer de reconstituer les élites, à chaque génération; elle juge d'ailleurs que la qualité de la formation, le développement des excellences dans l'école, est le moyen le moins injuste pour détecter les meilleurs. Alors que l'égalité est la valeur essentielle pour la logique égalitaire, la recherche de l'excellence, la sélection et la compétition pour développer les qualités intellectuelles sont les valeurs de la logique élitaire.

La manière dont on a construit le collège commun a consisté à ignorer les possibles contradictions entre les deux logiques à l'œuvre. En fait, les responsables, les observateurs du système scolaire, ont nié l'inévitabilité de la logique élitaire. Beaucoup d'études sur l'école vouent le collège à développer exclusivement une logique égalitaire : 
ainsi on supprimera les filières, mais la logique élitaire a établi de fait une hiérarchie d'un collège à l'autre, voire aussi la présence de ces lycées de centre-ville au fonctionnement élitiste. En refusant de prendre en compte la logique élitaire pour tenter de mettre plus de justice sociale dans l'accès aux élites, on a laissé se développer dans le système scolaire une logique élitiste de sélection sur la base des origines sociales, au détriment de la majorité de la population.

Les contradictions non gérées entre les deux logiques ont eu des effets déstabilisateurs sur une institution scolaire en porte-à-faux avec des valeurs républicaines, prônant l'égalité et, malgré cela, travaillée en fait par des processus de dévalorisation des enfants d'origine modeste. Le gros problème est aujourd'hui de sortir de la perversion qu'est la logique élitiste pour mettre plus de justice dans des processus élitaires réellement ouverts à toute population scolaire.

\section{3. Retour aux fondamentaux ?}

32 Au cours des années de déconstruction de l'école, une disjonction néfaste s'est produite: on a disjoint laïcité et république. La laïcité scolaire tendait à se réduire au culte des droits de l'homme, ceux-ci entendus dans un sens purement individualiste ${ }^{3}$. Et, à la limite, la totalité du Droit devait se limiter aux droits individuels. C'était l'ère de la société multiculturelle, le dogme du pluriel avait réponse à tout. Mais ces grands intellectuels généreux de tête méconnaissaient en leur belle âme que, dans une société, le pluriel a sens par rapport à un second pôle indispensable : l'unité. Le pluralisme culturel dans une société est pure fragmentation du social s'il ne s'équilibre pas dans des éléments communs, une culture commune qui maintient par-delà la pluralité l'unité d'une société. Quand celle-ci éclate, le pluriel n'a plus de sens : on a des mondes fermés, séparés. Or c'est bien dans la disjonction entre laïcité et République qu'on mutile celle-ci en en faisant le lien d'une pluralité qui ne s'équilibre plus dans une unité symbolique.

La question qui se pose à une société démocratique et laïque est fondamentalement: qu'est ce qui fait l'unité du social ? Étant laïque, cette société ne peut plus vivre et penser son unité autour d'une religion commune ou déterminante. Étant démocratique, cette société ne peut plus compter sur un pouvoir fort et dominateur pour contraindre à l'unité. Au bout du compte il s'agit là d'une société fragile. Certains avancent comme solution, comme ciment de l'unité de la société, la démocratie. Mais celle-ci règle la question d'un pouvoir contrôlé par le peuple dans la sphère politique d'une société déjà là. La preuve en est que, si la société explose, les procédures démocratiques sont bloquées. Pour qu'une démocratie advienne il faut déjà une société. D'autres font passer le principe de cohésion d'une société sur le terrain économique. Mais là encore, quand une société se casse, l'économie ne suffit pas à l'éviter.

C'est ici que nous retrouvons la nécessité du côté des fondamentaux : laïcité et République s'équilibrent. Bien entendu, on ne réduit pas ici la République aux trois mots qui la symbolisent souvent : liberté, égalité, fraternité. Après l'âge monarchique où l'unité se fit autour du corps du souverain, la République désigne ce corps collectif, ce sens global d'une société qui se fait sujet historique collectif comme nation. La république dit l'unité, ce n'est pas pour rien que les Français la disent indivisible. Elle se relie à l'espace, celui de la nation, et au temps, à travers les récits où elle assume sa propre advenue, et l'héritage des temps qui l'ont précédé. 

limportance que les fondateurs attachèrent à la morale laïque. Plusieurs causes ont joué au cours du XXe siècle pour aboutir en 1969 à la disparition de la morale à l'école laïque : " Après mai 68 un arrêté d'août 69 organise le tiers temps pédagogique à l'école primaire et compte la morale parmi les activités d'éveil. Le programme de morale ne verra jamais le jour. Après cette date, il ne reste plus à l'école élémentaire et au collège que l'éducation/instruction civique » (OGNIER, 1994). On ne peut ici qu'évoquer différents facteurs qui à travers le XXe siècle ont abouti à ce constat de carence. Pierre OGNIER mentionne l'opposition de l'Église, notamment aux « devoirs envers Dieu » mais aussi à la morale laïque. Dès la fin du XIXe est fait le constat d'un essoufflement de la morale scolaire. Quand la philosophie, le scientisme, la sociologie viennent au secours de l'enseignement de la morale, cela ne fait qu'accentuer le déclin. En 1923 disparaissent du programme les devoirs envers Dieu, et le vieux programme est affaibli. Si le courage des soldats en 1914/18 est relié par certains à la morale laïque, après 1918 l'immense désastre de cette guerre décourage la morale scolaire. En 1939 encore, note Pierre OGNIER, «l'inspiration initiale de la morale scolaire est encore bien vivante.» Pourquoi la disparition de l'éducation morale? Dans un premier temps on a insisté sur l'éducation civique... On pourrait souligner que le mouvement des idées dans le dernier tiers du XXe siècle était assez peu porteur, en général, pour une valorisation de la réflexion éthique...

\section{5. Aimer le politique} devant l'urgence d'une culture sur le politique. La question qui se pose à la démocratie est de savoir s'il y aura suffisamment de citoyens actifs, c'est-à-dire engagés dans une action collective, pour que la démocratie existe encore dans vingt ans. L'enjeu principal n'est pas ici l'acquisition de quelques connaissances factuelles. Bref, l'école laïque devrait transmettre l'amour de la chose politique. Car la démocratie (avec les libertés) ne survivra pas sans de tels citoyens. L'école devrait informer sur la nécessité du pluralisme 
des partis, sur l'urgence d'une présence dans ces partis de simples citoyens sans projet de carrière politique. Tout cela implique pour l'école d'assumer une véritable culture du politique. Il est insupportable que n'apparaisse pas à l'école la reconnaissance de la noblesse foncière de l'action politique. Que le mépris pour les politiques dans la société affleure à l'école, rien d'étonnant à cela. Mais l'école de la République a pour tâche d'opposer à une réalité dévalorisée le sens profondément humain et noble de l'action politique.

\section{6. Culture et sens}

Enfin, nous reviendrons sur ce progrès décisif qu'aura été l'effort pour ouvrir les disciplines scolaires à une connaissance des religions. Il ne s'agit pas seulement de permettre une meilleure compréhension entre des citoyens aux opinions diverses. Les religions, comme les philosophies et les arts, sont des domaines dans lesquels les humains s'efforcent d'élucider la question du sens: d'où vient l'humanité, où allons-nous? Comment répondre à la question : qui est l'humain?

41 Il n'est pas dans la compétence de l'école laïque de donner une réponse définitive à ces questions. Mais une culture vivante devrait proposer des moyens pour avancer dans la clarification de ces grandes interrogations. Quand on réfléchit sur le message d'une religion, sur les chemins qui sont proposés, on voit comment des humains ont mené cette recherche sur le sens. On peut discuter leurs méthodes. Mais on se place ainsi sur le terrain même d'une recherche qui peut évidemment prendre beaucoup de temps. De la même façon en philosophie, ce qui compte ce n'est pas de s'arrêter aux conclusions du penseur, de réduire sa pensée à des dogmes. L'important c'est, en l'accompagnant dans la démarche de sa pensée, d'apprendre soi-même à inventer sa propre démarche. La laïcité scolaire ne détient pas les réponses aux grandes questions, mais elle aurait tort de fuir devant les questions. Il lui revient d'apprendre aux élèves à aborder lucidement, intelligemment, ces questions, en se faisant aider par ceux qui nous ont précédés dans cette recherche.

\section{Au cœur de la crise, l'islam interpelle la laïcité.}

C'est dans les années 80, probablement au point extrême de déconstruction de la laïcité scolaire et de l'effacement de la jonction laïcité, école, République, que s'impose un événement qui va agir comme grand défi à la laïcité : la demande exprimée par l'islam de se voir reconnaître une juste place dans l'espace démocratique. Cette demande va se concentrer symboliquement sur le voile islamique, et symboliquement aussi, c'est vis-àvis de l'école, ce lieu vital de la laïcité républicaine, qu'elle va s'exprimer. On se souvient qu'à ce moment les familles des travailleurs émigrés comprennent que leur installation en France est sans retour. Du coup pour les familles de tradition musulmane surgit l'impératif de construire un islam dans la société française, dans la République laïque. C'est autour de la longue polémique sur le voile que va d'abord être posée, et fort mal, la question d'une entente possible entre l'islam et la laïcité.

Or nous sommes alors dans une période de grand flottement sur le sens de la laïcité à l'école, et sur l'idée de la République. Autour des années 90 , se référer à l'idée républicaine passe aux yeux de toute une partie de la classe intellectuelle comme une 
quasi allégeance aux idées de Jean-Marie LE PEN... L'incertitude sur la laïcité régnait aussi dans l'État. N'oublions pas que la grande fête du bicentenaire de la Révolution fut « droit de l'hommiste " et fort peu républicaine. Quand les premiers incidents sur le voile islamique se produisent à Creil à la rentrée 1989, le ministre de l'Éducation nationale, Lionel JosPin, au lieu de trouver dans la tradition républicaine de la laïcité scolaire les arguments pour légitimer les limites à l'ostensibilité des signes religieux, dégage en touche, se défausse en demandant au Conseil d'État de trancher. Or ce problème n'était pas une première. Au temps du Front populaire, le ministre Jean ZAY, confronté à l'exagération des expressions politiques dans l'école publique avait pris une circulaire fixant des limites, mais aussi bien sur le terrain politique que religieux. En fait, on demandait aux populations issues de l'émigration de consentir à s'intégrer, mais dans les institutions le flottement sur le sens de la laïcité et de la République justifiait en un sens une question : à quoi devons-nous nous intégrer? Les représentations régnantes sur la société multiculturelle, sur la réduction de l'idée de droits à ceux des individus rendaient difficilement perceptible l'identité certes particulière mais réelle cependant de cette société historique. Qu'elle ait été le berceau des déclarations des droits de l'homme, de portée universelle, il reste que l'universel ne définit pas seul l'identité d'une société. Rejoindre cette société c'est aussi accepter sa spécificité, sa particularité, son histoire.

En bref, une nouvelle religion frappe à la porte de la République... au moment même où celle-ci a perdu conscience d'elle-même, les écrits des élites le révèlent. Du coup, à travers la question posée à l'école, l'islam fait erreur sur les conditions de son intégration dans l'espace laïque et républicain. Il heurte le principe laïque de l'école en prétendant s'y imposer en corps, tous derrière un drapeau prêt à conquérir l'espace scolaire. C'est ainsi que, et pendant quinze ans, l'affaire du voile islamique va perturber l'école et aider en fait les courants islamistes à s'imposer comme porte-parole de l'islam. Dès lors que la volonté de la République n'était pas claire, dès lors que le sens même de la laïcité scolaire était confus, on en fut réduit à patauger dans les flottements d'une jurisprudence impossible à fixer.

45 L'enjeu dépassait largement la question des signes inacceptables dans l'école et de l'expression d'une conscience religieuse dans la classe. On était devant la revendication à la reconnaissance dans l'espace républicain d'une nouvelle religion. Dans les premières années du XXIe siècle, la question globale de l'islam dans la République devient enfin audible. Certes sur la question de la représentation de l'islam de France, Pierre Joxe avait auparavant lancé les bonnes pistes (1990) mais la question entre dans une phase définitive quand en $2002 \mathrm{~N}$. SARKOZY fait accoucher au forceps un Conseil français du culte musulman : la méthode est mauvaise, le ministre de l'intérieur viole allégrement la loi de 1905 qui interdit à l'État d'organiser autoritairement une religion, mais le progrès est évident dans la reconnaissance de l'islam comme fait collectif.

De plus, dès 2002, la mise en place de la commission STASI affronte la globalité des problèmes que pose l'application de la laïcité au XXIe siècle. Les solutions ne sont pas toutes viables, mais le signe est certain: la République n'a plus honte d'elle-même, le tournant est pris vers une redécouverte de liens serrés entre laïcité et République.

47 L'une des mesures adoptées à la quasi-unanimité par la commission STASI est la nécessité de légiférer sur la laïcité scolaire. L'argument décisif avait été qu'il fallait un texte législatif clair pour que la Cour européenne puisse trancher sur la conformité des décisions de justice avec les règles de droit régissant les religions en France. L'idée que cette loi était devenue nécessaire avait gagné l'appui de la majorité des politiques. 
Certains observateurs jugeaient qu'une telle loi serait inapplicable et accroîtrait l'ampleur des problèmes. Mais la rapidité avec laquelle la loi aura été acceptée dès la première année d'application ${ }^{4}$ semble donner raison à ceux qui estimaient que la cause des polémiques était largement dans le flottement de la légalité républicaine. Cet arrêt soudain de la militance organisée pour le voile dès la mise en place de la loi plaide également pour la thèse de ceux qui voyaient dans la multiplication des cas de jeunes filles voilées à l'école un phénomène fort peu spontané mais provoqué par des mouvements, des officines, voire des versements d'argent, situation qu'on retrouve ultérieurement avec la burqa.

\section{Vers une refondation de la laïcité scolaire et de l'école républicaine}

Parler de refondation ne signifie pas croire qu'on peut revenir un siècle en arrière. Cependant on peut penser que l'effacement de la laïcité et de l'école républicaine n'avait rien de fatal et de définitif. L'idée est qu'il est nécessaire à la société moderne et démocratique de demeurer capable de réinstitutionnaliser son espace commun d'une génération à l'autre, que cette société vouée à l'accueil du pluralisme en son sein, et même de divisions, a besoin d'une laïcité vivante pour ne pas éclater. De plus, société dont l'unité symbolique ne pourra plus être fondée sur le religieux, sauf à tomber dans les régressions totalitaires, cette démocratie a plus que jamais besoin de s'enraciner dans le meilleur de la tradition républicaine. Ce travail de réinstitutionnalisation n'est possible que par l'éducation confiée à l'école républicaine.

On nous objecte: mais si les données de la sociologie montrent que tout cela se fait de plus en plus mal, si même la violence ne peut être maintenue à l'extérieur de l'institution scolaire, comment réinstitutionnaliser l'école laïque et républicaine?

Un regard sur les origines montre à l'œuvre dans la naissance de l'école laïque et républicaine une forte volonté politique. Il n'était pas fatal que l'institution scolaire parvînt à se situer comme institution essentielle de la République. La condition première pour que l'institution scolaire puisse rejoindre son essence républicaine et laïque est que la république en ait la volonté politique. On peut noter qu'il y ait déjà eu certains exemples de cette volonté : nous reviendrons sur la loi de mars 2004, mais aussi sur l'ouverture de l'école au fait religieux.

\section{1. Le tournant de la loi sur les signes ostensibles}

51 C'est le 15 mars 2004 qu'est finalement votée la loi «encadrant, en application du principe de laïcité, le port des signes et de tenues manifestant une appartenance religieuse dans les écoles collèges et lycées publics ». L'article principal est bref et net: «Dans les écoles, les collèges et les lycées publics le port de signes ou tenues par lesquels les élèves manifestent ostensiblement une appartenance religieuse est interdit ». Il est précisé que cette loi sera applicable dès la rentrée de septembre 2004 et qu'elle fera l'objet "d'une évaluation un an après son entrée en vigueur » (articles 3 et 4). Dès mai 2004, une circulaire d'application signée du ministre de l'Éducation nationale François FILLON est adressée aux services de l'Éducation nationale. 

de la loi de mars 2004. Il y est rappelé que cette loi est prise « en application du principe constitutionnel de laïcité qui est un des fondements de l'école publique. Ce principe, fruit d'une longue histoire, repose sur le respect de la liberté de conscience et sur l'affirmation des valeurs communes qui fondent l'unité nationale par-delà les appartenances particulières. L'école a pour mission de transmettre les valeurs de la République parmi lesquelles l'égale dignité de tous les êtres humains, l'égalité entre les hommes et les femmes et la liberté de chacun y compris dans le choix de son mode de vie. Il appartient à l'école de faire vivre ces valeurs, de développer et de conforter le libre arbitre de chacun, de garantir l'égalité entre les élèves et de promouvoir une fraternité ouverte à tous. En protégeant l'école des revendications communautaires, la loi conforte son rôle en faveur d'un vouloir vivre ensemble. " le ton et la force d'une véritable charte de la laïcité scolaire. On peut affirmer sans risque d'erreur qu'il n'y avait jamais eu depuis les textes fondateurs une telle explicitation du sens de la laïcité scolaire. On y trouve l'expression de la mission essentielle de l'école dans la transmission et l'exigence de "faire vivre » les valeurs communes ou les « valeurs de la République ». On avait perdu l'habitude d'entendre ce rappel de la mission de l'école en faveur de vivre ensemble, et ceci à travers une exigence éthique. On lit ici une définition de la mission éducative de l'école qui sonne comme un retour aux fondamentaux de l'école laïque. Le même ton est présent dans le discours de Jacques CHIRAC après le rapport STASI. Certes, il est bien question de préciser l'application d'un texte sur les signes religieux, mais il faut insister sur le détail des rappels pratiques concernant l'exigence de la laïcité scolaire. Relevons : dénonciation du racisme, du sexisme, de «toutes les formes de violence faite à un individu en raison de son appartenance réelle ou supposée à un groupe ethnique et religieux ». Le texte exclut aussi : « Tout propos, tout comportement qui réduit l'autre à une appartenance religieuse ou ethnique, à une nationalité (actuelle ou d'origine)...»

54 L'exigence de la laïcité scolaire comporte aussi la connaissance des faits religieux. La liste des obligations de la pratique de cette laïcité est complète : un élève n'a pas le droit de s'opposer à un enseignement en raison de ses convictions religieuses, il n'a pas le droit «de contester le droit d'un professeur parce que c'est un homme ou une femme, d'enseigner certaines matières ou le droit d'une personne n'appartenant pas à leur confession de faire une présentation de tel ou tel fait historique ou religieux ».

55 Les règles d'assiduité sont longuement rappelées. Ainsi de l'obligation d'assister à tous les cours: «les convictions religieuses ne peuvent justifier un absentéisme sélectif par exemple, en éducation physique et sportive ou en sciences de la vie et de la terre »; « les convictions religieuses ne sauraient non plus être opposées à l'obligation d'assiduité ni aux modalités d'un examen"; est refusée aussi l'absence systématique un jour de la semaine ${ }^{5}$.

56 En plus du rappel des règles fondamentales de la laïcité scolaire, ce texte constitue une remarquable synthèse sur la pratique de cette laïcité aujourd'hui. On peut considérer qu'il s'agit là, dans le sillage de la loi sur les signes religieux, d'un véritable tournant, d'un acte de ré institutionnalisation de la laïcité et de l'école. Il ne s'agit aucunement d'un durcissement de la laïcité scolaire. Rappelons-le, dans les années d'après 68, et notamment les années 80 , on assistait à une sorte d'effacement de la laïcité scolaire. Se référer à la laïcité, voire aux valeurs républicaines, cela sonnait ringard. L'époque était au

Tréma, 37 | 2012 
multiculturalisme, au triomphe de l'individualisme. Le fait est que c'est à cette période que va s'exprimer l'interpellation de l'islam à la laïcité. Mais notons-le, c'est aussi au moment où tendait à se réaffirmer la valeur de la laïcité, où celle-ci était redevenue un thème légitime pour des livres, des articles, des dossiers, des débats, que va être votée la loi de mars 2004.

\section{2. Les religions dans la culture scolaire} sur l'école catholique s'était apaisée. De ce grand débat public, il allait se dégager des pistes sur ce que devrait être cet enseignement: il ne serait pas confessionnel mais culturel et laïque, c'est-à-dire distinct de la catéchèse. Fallait-il s'orienter vers une discipline autonome, ou demander à diverses disciplines scolaires d'aborder avec leurs moyens propres, leurs compétences culturelles, cette connaissance des faits religieux? Finalement cette solution pluridisciplinaire s'imposa. Une discipline prit une certaine avance, l'Histoire. Plusieurs responsables de cette discipline furent moteurs. Dès 1989, dans un rapport qui fit date, le recteur Philippe JOUTARD prenait position contre l'idée d'une discipline autonome. Le poids excessif des historiens était également manifeste dans un colloque qui marqua profondément le débat ${ }^{7}$. À partir de 1995, diverses réformes dans les programmes (histoire, français...) se suivent. Après les années d'immobilisme, sous l'impulsion de Jack LANG, ministre de l'Éducation nationale, le rapport dirigé par Régis DEBRAY (mars 2002) constitua une relance de la question, mais à part la création de l'Institut européen des sciences religieuses qui devait animer la formation des enseignants, le rapport DEBRAY ne donna lieu à aucune initiative de relance, malgré l'immense qualité des arguments développés dans ce rapport.

Parmi les bonnes mesures proposées, relevons une proposition pour un minimum de formation des futurs enseignants en seconde année d'IUFM. La suppression de ces instituts a tranché par le néant la formation des futurs enseignants à l'approche du fait religieux. Une mesure importante passa presque inaperçue. Philippe JOUTARD, artisan du nouveau programme dans l'école élémentaire, y avait intégré un minimum d'ouverture aux religions : «Des ouvertures culturelles sont prévues au cours des trois dernières années du primaire. L'époque antique conduira à une première approche du monothéisme à travers la christianisation de l'Europe. En CM1 les enfants découvriront la période médiévale, où le christianisme imprègne l'ensemble des activités du vieux continent, la culture des abbayes et des cathédrales. Un sujet sur la méditerranée introduira l'islam: les apports culturels et les conflits autour des lieux saints. Puis l'époque moderne, marquée par la Renaissance et le protestantisme ${ }^{8}$ ".

60 Depuis le début des années 2000, la prise en compte de l'importance d'un développement de la connaissance des religions dans la culture scolaire comme nouveauté laïque essentielle a été largement oubliée. Le minimum qui s'imposerait aujourd'hui, ce serait de faire un grand bilan de ce qui se fait, de ce qui ne se fait pas. Là où les programmes ont bougé, il y a présence de l'étude des religions. Mais quels sont les freins qui jouent négativement? Quels sont les besoins des enseignants? Quelles sont les insuffisances des programmes? Tant de questions sont à l'abandon! Nos gouvernements ne devraient-ils 
pas méditer les propos d'un homme, Ferdinand BuIsson, qu'on ne peut soupçonner de laxisme en matière de laïcité de l'école : pour l'éducation d'un enfant qui doit devenir un homme, il est bon qu'il ait été tour à tour mis en contact avec les strophes enflammées des prophètes d'Israël, avec les philosophes et les poètes grecs... Il serait bon qu'on lui fasse connaitre les plus belles pages de l'Évangile, comme celle de Marc AURèLE, qu'il ait feuilleté, comme Michelet, toutes les bibles de l'humanité » (dans La Foi laïque, 1908). Pour ceux qui craignent que les limites imposées aux signes religieux correspondent à une fermeture de l'école au fait religieux, il importe de rappeler cette présence culturelle des religions dans l'espace scolaire. Du coup, on est sorti de ce que j'ai parfois appelé la laïcité-silence. J'entends par là que pour certains, la vraie laïcité scolaire aurait signifié un absolu silence sur la réalité des religions dans l'école. Or le fait religieux étant l'objet d'étude, il est dans la logique de la laïcité scolaire que les élèves puissent exprimer librement dans la classe leurs questions touchant au domaine religieux, leurs positions personnelles. La fonction d'un maître laïque étant alors d'assurer un équilibre dans l'expression de chacun, un respect strict des contradictions, des divergences fortes. Et ceci est possible, nous pouvons en témoigner, pourvu que tous aient reconnu que l'autorité du maître est garante du droit de chacun à sa parole.

\section{3. Signes de ressaisissement}

Quant aux réactions nécessaires pour lutter contre l'ensemble des entorses à la laïcité et aux valeurs républicaines dans l'école, nous reprendrons ici quelques recommandations fortes qui concluent le rapport oBIN cité plus haut. L'auteur signale des évolutions inquiétantes, qui font que dans les établissements scolaires le racisme anti-maghrébin a été supplanté par le racisme anti-juif: «Il est en effet sous nos yeux une stupéfiante et cruelle réalité : en France les enfants juifs, et ils sont les seuls dans ce cas, ne peuvent plus de nos jours être scolarisés dans n'importe quel établissement »(OBIN, 2004, p. 23).

La première proposition du rapport demande de «tout faire pour développer la mixité sociale dans les établissements scolaires » (p. 32) (par exemple en remodelant les cantons de sectorisation de certaines zones). Le rapport propose aussi de «former et aider les professeurs à répondre aux contestations de leur enseignement » (p. 32) et d'inciter « les inspecteurs territoriaux à s'intéresser de près [à ces contestations] » (p. 33) (à cet égard, il $\mathrm{y}$ a une critique de la formation des enseignants aux contestations religieuses des élèves).

Enfin, Jean-Pierre OBIN suggère de « piloter plus fermement à tous les niveaux » (p. 33). À cet égard, il donne en exemples les établissements " où l'on ne tolère pas l'intolérable " (p. 33), où l'indifférence et la pusillanimité ne sont pas de mise lorsqu'il s'agit de menaces de violences notamment à l'encontre des plus faibles et des filles, de prosélytisme agressif, de racisme ou d'antisémitisme ». Il prône l'intransigeance, la communication sur les faits, la fermeté sur les principes républicains. Il demande aux instances hiérarchiques d'être bien informées. Il dénonce les établissements "sans conflits ». "Pour trop de responsables, un établissement sans conflits est un établissement sans problème. Au contraire, dit-il, ce sont des établissements où l'on a cédé sur l'essentiel » (p. 34). Cette attitude de lucidité et de courage va à l'encontre d'une culture du laxisme, de l'évitement : ne pas avoir de conflits pour être bien vu de la hiérarchie.

En fait, ce qui ressort d'un tel document c'est l'urgence d'un ressaisissement de toute la structure Éducation nationale, en incluant bien entendu le sommet, les responsables politiques. Les données d'Emmanuel BRENNER et de Jean-Pierre OBIN datent d'avant la mise 
en application de la loi sur le voile. Ne peut-on penser que la fermeté de ce texte, et de sa circulaire d'application a pu jouer dans le sens d'un «rétrécissement des territoires perdus de la République » (BRENNER, 2004) ? La chose est claire, sans un renouvellement de la volonté politique de faire vivre l'institution, de la ré-institutionnaliser, l'école de la République, qui est au fondement de l'avenir de la démocratie, pourrait aller vers un effondrement irréversible. Cette volonté politique suppose d'avoir le courage d'imposer des limites claires. C'est ainsi que la récente circulaire du ministère de l'Intérieur sur les cantines va dans le bon sens: "Le fait de prévoir des menus en raison de pratiques confessionnelles ne constitue ni un droit pour les usagers ni une obligation pour les collectivités ».

\section{4. Re-sacraliser d'anciennes fêtes religieuses ?}

L'application de la laïcité scolaire rencontre diverses revendications concernant le calendrier scolaire et notamment les jours fériés. L'origine chrétienne de certaines dates fait problème à certains, au regard du principe même de laïcité. Du coup il a été parfois « suggéré » d'adopter quasiment le principe d'un jour férié par religion : tel jour pour une fête juive, tel autre pour une fête musulmane. Cette revendication suscita il y a quelques années un article fort éclairant de Yolaine DILAS-ROCHERIEUx (directrice du département de sociologie de l'université Paris 10). Partant du principe que ces fêtes à l'origine chrétienne se sont en fait laïcisées, elle écrit : « Mais introduire une journée fériée propre aux différentes religions reviendrait en fait à redonner au calendrier issu de la chrétienté un caractère sacré, à inverser le processus de sécularisation » (DILAS-ROCHERIEUX, 2005, p. 81). Certes, note l'auteur, il y a ainsi une inégalité entre des religions anciennement présentes et qui ont laissé des marques inscrites dans la culture commune et des religions plus récemment présentes: "Or toute société a son histoire et personne ne peut envisager d'y renoncer ou d'en élaguer certains épisodes mêmes s'ils sont négatifs sous prétexte de diversité culturelle et religieuse. Les dates des diverses célébrations renvoient bien évidemment à un passé judéo-chrétien et à des événements fondateurs ou libérateurs à l'instar du 14 juillet 1789 ou de l'armistice du 8 mai 1945 » (p. 81). Ce qui est en cause c'est l'inégalité des marques inscrites dans l'héritage culturel et le cadre de vie. Gilles BERNHEIM va aussi dans ce sens quand il s'agit de l'égalité entre les diverses religions : «La loi de 1905 n'est pas censée assurer aux cultes une égalité de moyens mais une égalité de liberté d'exercice. Cette exigence d'égalité peut s'exercer au détriment d'autres libertés également garanties par la constitution [...]. Dans la pratique le financement et l'entretien de nouveaux lieux de cultes qui viennent s'ajouter aux obligations d'entretien touchant le patrimoine qui date d'avant 1905, peut arriver à gravement peser sur le budget de certaines communes, et ce au détriment d'autres équipements nécessaires et accessibles à tous » (BERNHEIM, 2005).

De plus, l'auteur fait remarquer que ces lieux de culte d'avant 1905 ne sont pas seulement des locaux utilisés par une confession : "Car ce qui est vu comme un "parc d'églises" n'est autre que le patrimoine historique, religieux, social et culturel de la France ». De la même façon que l'on ne peut ignorer plus d'un millénaire d'histoire, on ne peut revisiter cette histoire au gré des revendications des uns et des autres ». Pour éclairer cette remarque, Gilles BERNHEIM cite un texte très significatif du grand juriste Jean RIVERO : « Les mœurs même coupées de leurs racines religieuses ont prévalu sur la logique intégrale de la séparation. Par là s'explique aussi le fait qu'en dépit de l'égalité de principe entre toutes 
les religions, l'État entretienne avec les cultes traditionnels des rapports plus suivis qu'avec les cultes nouveaux [...]. Laisser dépérir les églises eût été priver le paysage français d'un de ses éléments essentiels et rompre avec la tradition antérieure » (Jean RIVERO, cité Par BERNHEIM, 2005).

L'argument ici évoqué est de grand poids: il dit en somme que les religions traditionnelles ont contribué à modeler les grands héritages culturels qui caractérisent ce pays. Ceci est vrai des divers pays : «La sphère publique a été façonnée par l'histoire politique, économique, culturelle et religieuse particulière de chaque pays ».

La conséquence tirée de cela est que, pour Gilles BERNHEIM, « la réception de l'islam par la France ne peut se faire sans l'acceptation des valeurs françaises par l'islam ». Et l'auteur ajoute que toutes les grandes traditions religieuses, protestantisme, judaïsme, catholicisme ont dû faire un effort pour s'intégrer à la République, "pour intégrer au sens le plus profond du terme l'histoire de la France et contribuer à forger son identité particulière ".

Il y a là, dans les rapports entre le christianisme et la laïcité, un thème trop peu réfléchi : il y a en France une forme de christianisme culturel, c'est-à-dire des marques chrétiennes qui interviennent sans présupposer aucunement la foi - marques inscrites dans les rythmes de vie, dans les monuments, la langue des grands héritages de la culture nationale. Poser comme condition à la laïcité, notamment scolaire, d'avoir à gommer systématiquement ces marques, ce serait déstructurer la culture française et même européenne.

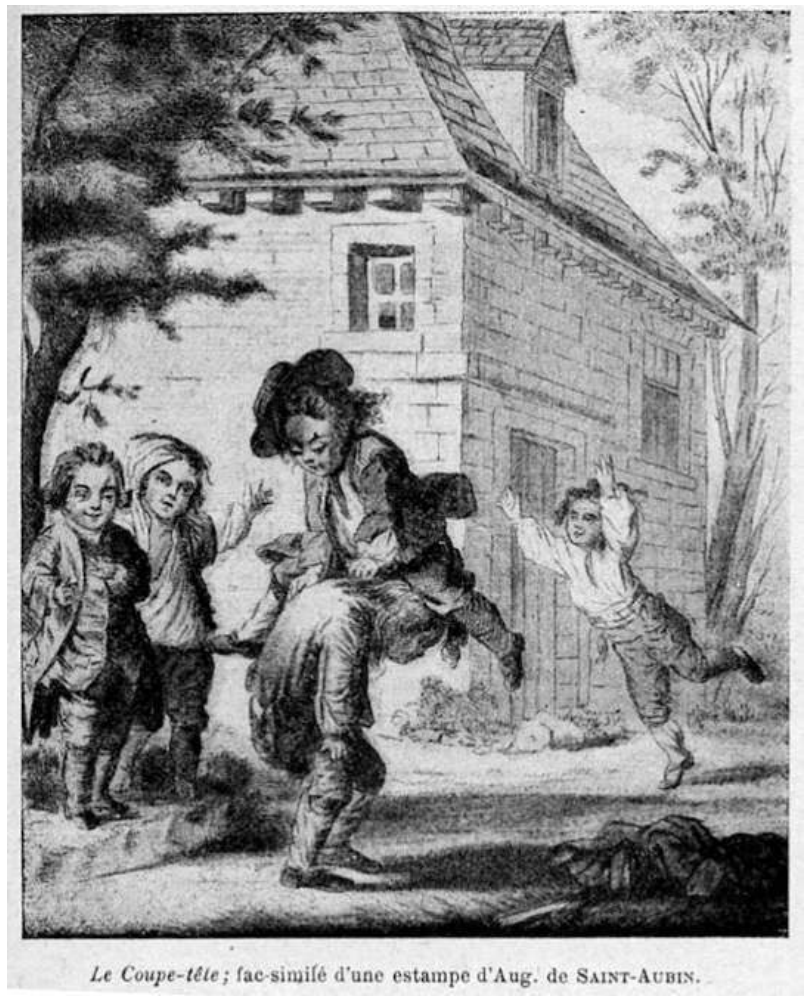

Livre de lecture et de morale, cours moyen et supérieur, E. DEVINAT, Paris, Librairie LAROUSSE, p. 59. 


\section{Le Mauvais camarade,}

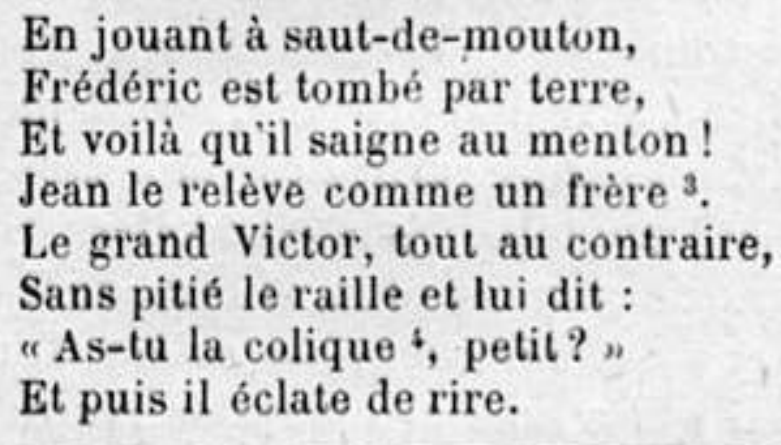

Livre de lecture et de morale, cours moyen et supérieur, E. DEVINAT, Paris, Librairie LAROUSSE, p. 60.

\section{BIBLIOGRAPHIE}

ARENDT (Hannah), La Crise de la culture. Huit exercices de pensée politique (1968), trad. fr. sous la direction de Patrick LÉvY, Paris, FolIO-GALLIMARD, 2007.

BAUBÉROT (Jean), La morale läque contre l'ordre moral, Paris, éd. du SEUIL, 1997.

BERNHEIM (Gilles), « Faut-il réviser la loi de 1905 ? », Le Figaro du 19-20 février 2005.

BRENNER (Emmanuel) (dir.), Les Territoires perdus de la République, Paris, Mille et une nuits, 2004. COQ (Guy), La laïcité, principe universel, Paris, LE fÉLIN, 2005.

DILAS-ROCHERIEUX (Yolaine), « Choses vues et entendues à l'Université », in SEKSIG (Alain) (dir.), Laïcité : les cent ans d'une idée neuve. I. À l'école, revue Hommes et migration, 꾸1258, novembredécembre 2005. Dossier disponible en ligne : http://www.hommes-et-migrations.fr/docannexe/file/5492/dossier_1258_dossier_1258.pdf GAUCHET (Marcel), La Démocratie d'une crise à l'autre, Paris, 2007, éd. Cécile DEFAUT. OBIN (Jean-Pierre), Les signes et manifestations d'appartenance religieuse dans les établissements scolaires, rapport de l'IGEN, 2004.

OGNIER (Pierre), Histoire de la laïcité, CRDP de Franche Comté, 1994 (collectif avec Guy GAUTHIER, Louis LEGRAND et Jean BAUBÉROT).

PROST (Antoine), L’Enseignement en France, 1800-1967, Paris, éd. Armand colin, 1968.

\section{NOTES}

1. Ici, comme en divers endroits, je m'appuie sur les travaux de Pierre OGNIER sur la morale à l'école. 
2. Circulaire du 17 novembre 1883.

3. Renvoyons une fois pour toute aux travaux éclairants de Marcel GAUCHET (notamment GAUCHET, 2007).

4. Voir le rapport sur l'application de la loi établi par Hanifa CHÉRIFI à l'issue de la première année.

5. Dans beaucoup de ces rappels concernant la laïcité à l'école, on peut lire en fait la réaction énergique de l'Éducation nationale contre les dérives dénoncées dans le rapport de J.-P. OBIN.

6. Congrès de Montpellier 1982.

7. Colloque de Besançon, 20 et 21 novembre 1991.

8. Philippe JOUTARD. Pour une synthèse sur le sujet, voir COQ, 2005, p. 119 à 161.

\section{RÉSUMÉS}

Trois grandes orientations caractérisent la laïcité en France dès les débuts; d'une part, le catholicisme n'est plus reconnu comme religion d'État, ce dernier se marquant par sa neutralité, d'autre part l'État garantit le libre exercice des culte ce qui signifie que la République est indépendante de toute confession religieuse, et ce qui ouvre également à une éthique de la diversité. Enfin le lien social ainsi institué ne dépend plus d'une altérité (divine, naturelle, immanente...). L'ensemble de la société est remis à la responsabilité des citoyens, c'est pourquoi la laïcité comme principe d'autonomie de la société débouche sur le principe démocratique. Ainsi, nous verrons comment l'école hérite de l'ensemble de ces dimensions et comment, loin d'être un simple espace d'application du principe de laïcité, elle est cet espace fragile au sein duquel s'explicite une part du sens même du principe de laïcité.

Three main orientations characterize Secularism in France from the beginning. On the one hand, Catholicism is no longer recognized as state religion, marking its neutrality. On the other hand the State guarantees freedom of worship which means that the Republic is independent of any religious denomination, and which also opens to an ethic of diversity. Finally this social bond no longer depends on an otherness (divine, natural, immanent ...). The whole society is given the responsibility of citizens. Secularism as a principle of autonomy of society leads to the democratic principle. We will see how the school inherits all these dimensions and how, far from being a mere space of the principle of secularism, it is this fragile area in which is working a part of the very meaning of the principle of secularism.

\section{INDEX}

Mots-clés : autonomie, démocratie, école, principe de laïcité

Keywords : autonomy, democracy, principle of secularism, school 


\section{AUTEUR}

\section{GUY COQ}

Agrégé de philosophie, membre de la rédaction de la revue Esprit 